\title{
Association of the type of trauma, occurrence of bone bruise, fracture and joint effusion with the injury to the menisci and ligaments in MRI of knee trauma
}

\author{
Sina Pezeshki ${ }^{1}$ \\ Thomas J. Vogl' \\ Mohammad Zakaria Pezeshki² \\ Mohammad Hossein Daghighi ${ }^{3}$ \\ Masoud Pourisa ${ }^{3}$ \\ 1 Department of Diagnostic and Interventional Radiol- \\ ogy, Johann Wolfgang Goethe University Frankfurt, \\ Frankfurt, Germany \\ 2 Department of Community Medicine, Tabriz \\ University of Medical Sciences, Tabriz, Iran \\ 3 Department of Radiology, Tabriz University of \\ Medical Sciences, Tabriz, Iran
}

Corresponding author:

Mohammad Zakaria Pezeshki

Department of Community Medicine, Tabriz Universi-

ty of Medical Sciences

Golgasht Avenue

5154753566 Tabriz, Iran

E-mail: zakaria.pezeshki@gmail.com

\section{Summary}

Background: magnetic resonance imaging (MRI) as a noninvasive diagnostic tool may help clinicians in the evaluation of injuries to menisci and ligaments.

Purpose: this study assessed the associations between type of trauma to knee joint, bone bruise, fracture and pathological joint effusion with injuries to menisci and ligaments of knee joint.

Methods: we reviewed knee joint MRI of 175 patients aged less than $\mathbf{4 5}$ years old who were referred to MRI center of our University.

Results: statistical analysis showed that tearing of medial meniscus (MM) is significantly more common in sport related trauma $(p=0.045)$ but tearing of medial collateral ligament (MCL) is significantly more common in non-sport related trauma $(p=0.005)$. Existence of bone bruise in knee MRI is negatively associated with tearing of medial meniscus (MM) $(p=0.004)$ and positively associated with tearing of anterior cruciate ligament $(A C L)(p=0.00047)$ and medial collateral ligament (MCL) $(p=0.0001)$. Existence of fracture is associated with decreased risk of the tearing of $A C L$ and $M M(p=0.04, p=0.001$ respectively). Pathologic joint effusion is significantly more common in $A C L$ and MCL tearing $(p=0.0001, p=0.004$ respectively).

Conclusions: as diagnostic clues, bone bruise, fracture and joint effusion may help radiologists for better assessment of injury to menisci and ligaments in MRI of patients with knee trauma.

KEY WORDS: magnetic resonance imaging, knee joint, ligaments.

\section{Introduction}

Magnetic resonance imaging (MRI) is used more commonly in the knee trauma comparing with other joints and is an excellent diagnostic tool that may help clinicians in the evaluation of injuries to menisci and ligaments, osseous structures, articular surfaces and tendon ${ }^{1-9}$. It plays an important role in clinical decision making 6,7 .

Bone bruise or contusion in the knee was described for the first time by Yao and Lee in $1988^{10}$. Bone bruise is considered as a microfracture ${ }^{10,11}$. For at least a decade the terms "bone bruise" and "bone marrow edema" have been known and are used synonymously ${ }^{12}$. It demonstrates a diffuse or localized decreased signal intensity on proton-density or T1weighted images and increased signal intensity on T2-weighted images, at the short tau inversion recovery sequence (STIR) or fat-suppressed MRI sequences $^{11-13}$. This appearance is thought to represent areas of hemorrhage, edema, or infarction secondary to trabecular microfractures that may all contribute to the marrow signal alterations ${ }^{11,13}$. Areas of bone contusion in MRI that help to understand the mechanism of injury, predict and confirm the presence of capsule ligamentous injuries ${ }^{9}$. The most common traumatic causes of knee effusion are ligamentous, osseous and meniscal injuries, and overuse syndromes ${ }^{14}$.

As much as the Authors know, there isn't any research about the association between fracture around knee joint, joint effusion on one hand and pattern of injury to the menisci and ligaments on the other hand. The main aim of this study is to assess if type of trauma, bone bruise, fracture and joint effusion are associated with the distinguished pattern of injury to menisci and ligaments. These associations may help radiologists to make more accurate diagnosis. 
This study assessed the associations between type of trauma to knee joint, bone bruise, fracture and pathological joint effusion with injuries to menisci and ligaments of knee joint.

\section{Materials and methods}

Sample. As a consecutive sampling 175 patients with knee trauma who were referred to MRI center of our university were recruited. The study lasted one year. A radiologist reviewed the MRI of the patients. The age of the patients were less than 45 years (range: $18-45)$. One hundred forty nine $(85.1 \%)$ were males and $26(14.9 \%)$ were females. Before MRI exam we asked patients about their age, type of trauma (sport related, non-sport related), time of trauma, and history of previous knee injury or surgery of knee. The age limitation in this study was because degenerative changes (degenerative meniscal tears) after the age of 45 years may bias our research finding. We included patients who were within 1 month of an acute knee injury when referred to our center. The time of 1 month was arbitrarily selected to define an acute injury. All patients with previous knee injuries or previous knee surgery were excluded. The study was approved by the ethical committee of our University.

MRI technique. The examination was performed with 0.3 T (Hitachi, Airis 2, Japan) using sagittal and coronal T1\&T2 weighted in phase and out of phase as well as fat suppression, fluid suppression, gradient echo images and axial T2 weighted spin echo images. The total imaging time of the standard protocol, including the initial survey sequence was $20 \mathrm{~min}$. The patient is usually imaged in the supine position with the knee nearly or fully extended and slightly rotated externally $10^{\circ}$ to align the anterior cruciate ligament parallel to the sagittal plane for better visualization.

Diagnostic criteria. Bone bruise was identified as an area of abnormal high signal intensity in the subchondral bone or marrow on the T2-weighted fat suppressed images. Menisci were classified as intact or torn. Meniscal tears were radiologically graded from I to IV. Grades III and IV were considered as "tear" group. As Grade I and II of tears may be related to meniscal degeneration so we categorized them with "Non tear" group.

Cruciate ligaments and collateral ligaments were dichotomized as normal or partial/total rupture.

Statistical test. All analyses were performed using SPSS (version 13.0.1, SPSS Inc, Chicago, III). Chi squ are test was used to test the associations. Logistic regression analysis was used for controlling confounding variables. The level of significance was fixed at $p<0.05$. The design and report of this study is according to the standard of the Muscles, Ligaments and Tendons Journal ${ }^{15}$.

\section{Results}

In this study 123 out of 175 (70.3\%) had non-sport related trauma and 52 out of $175(29.7 \%)$ had sport related trauma. Regarding the association between type of trauma with injury to menisci and ligaments we found that tearing of medial meniscus (MM) is significantly more common in sport related trauma $(p=$ 0.045 ) but tearing of medial collateral ligament ( $M C L$ ) is significantly more common in non-sport related trauma $(p=0.005)$.

In this study 54 patients out of 175 had bone bruise (30.9\%). As Table 1 shows, the existence of bone bruise is associated with decreased risk of tearing of medial meniscus (MM) and increased risk of the tearing of anterior cruciate ligament $(A C L)$ and medial collateral ligament (MCL) but has not any correlation with tearing of other ligaments and lateral meniscus (LM). Figure $1 \mathrm{~A}$ shows the MRI of a 21-year-old male patient with bone bruise as well as tear of MCL and Figure 1D shows the association of bone bruise with tear of $A C L$ in same patient. Also Figure $1 \mathrm{~B}$ shows normal MM in same patient.

Twenty one out of 175 patients had fracture (12\%). As Table 2 shows existence of fracture is associated with decreased risk of tearing of ACL and MM (Fig. 2 A-C) but has not any correlation with tearing of other ligaments and LM.

Table 1. Association of presence or absence of bone bruises with injury to the menisci and ligaments.

\begin{tabular}{llll}
\hline & No bone bruise & Bone bruise & p value \\
\hline $\mathrm{n}$ & 121 & 54 & \\
$\mathrm{ACL}$ tear & $32(26.40)$ & $29(53.70)$ & 0.00047 \\
$\mathrm{PCL}$ tear & $4(3.30)$ & $4(7.40)$ & 0.25 \\
MCL tear & $16(13.20)$ & $21(38.90)$ & 0.0001 \\
LCL tear & $3(2.50)$ & $5(9.30)$ & 0.11 \\
MM tear & $44(36.40)$ & $8(14.80)$ & 0.004 \\
LM tear & $34(28.10)$ & $13(24.10)$ & 0.58 \\
\hline
\end{tabular}

$\overline{\mathrm{ACL}}$, anterior cruciate ligament; $\mathrm{PCL}$, posterior cruciate ligament; $\mathrm{MCL}$, medial collateral ligament; $\mathrm{LCL}$, lateral collateral ligament; $\mathrm{MM}, \mathrm{medial}$ meniscus; LM, lateral meniscus. 
Association of the type of trauma, occurrence of bone bruise, fracture and joint effusion with the injury to the menisci and ligaments in MRI of knee trauma

Table 2. Association of presence or absence of fracture with injury to the menisci and ligaments.

\begin{tabular}{llll}
\hline & No fracture & fracture & p value \\
\hline $\mathrm{n}$ & 154 & 21 & \\
$\mathrm{ACL}$ tear & $80(51.90)$ & $6(28.60)$ & 0.04 \\
PCL tear & $7(4.50)$ & $1(4.80)$ & 1 \\
MCL tear & $34(22.10)$ & $3(14.30)$ & 0.57 \\
LCL tear & $8(5.20)$ & $0(0.0)$ & 0.60 \\
MM tear & $52(33.8)$ & $0(0.0)$ & 0.001 \\
LM tear & $43(27.90)$ & $4(19.0)$ & 0.39 \\
\hline
\end{tabular}

$\mathrm{ACL}$, anterior cruciate ligament; $\mathrm{PCL}$, posterior cruciate ligament; $\mathrm{MCL}$, medial collateral ligament; $\mathrm{LCL}$, lateral collateral ligament; $\mathrm{MM}$, medial meniscus; LM, lateral meniscus.

Table 3. Association of presence or absence of joint effusion with injury to the menisci and ligaments.

\begin{tabular}{llll}
\hline & No joint effusion & joint effusion & p value \\
\hline $\mathrm{N}$ & 93 & 82 & \\
ACL tear & $33(35.50)$ & $53(64.60)$ & 0.0001 \\
PCL tear & $2(2.20)$ & $6(7.30)$ & 0.15 \\
MCL tear & $12(12.90)$ & $25(30.50)$ & 0.004 \\
LCL tear & $3(3.20)$ & $5(6.10)$ & 0.48 \\
MM tear & $25(26.90)$ & $27(32.90)$ & 0.38 \\
LM tear & $21(22.60)$ & $26(31.70)$ & 0.17 \\
\hline
\end{tabular}

$\widehat{\mathrm{ACL}}$, anterior cruciate ligament; $\mathrm{PCL}$, posterior cruciate ligament; $\mathrm{MCL}$, medial collateral ligament; $\mathrm{LCL}$, lateral collateral ligament; $\mathrm{MM}$, medial meniscus; LM, lateral meniscus.

Eighty two out of 175 patients had joint effusion $(53.1 \%)$. As Table 3 shows pathologic joint effusion is significantly more common in tearing of $A C L$ and MCL (Fig. 1A-D). In patients with knee injury, tearing of MCL may coincide with tearing of ACL. The association between tearing of MCL and joint effusion may be confounded with ACL tearing. After controlling the effect of $A C L$ the association between $M C L$ and joint effusion didn't loss its significance $(p=0.09)$.

\section{Discussion}

This study found few significant associations between occurrence of bone bruise, fracture and joint effusion on one hand and injury to the menisci and ligaments on the other hand in patients with knee trauma who were referred to the MRI center of our University.

Type of trauma. We divided type of knee trauma into two groups: sport related and non-sport related. The higher prevalence of MM tearing in sport related trauma and MCL tearing in non-sport related trauma in our study may be related to the mechanisms of injury. We didn't find any study about association between type of trauma and injury to the menisci and ligaments.
Bone bruise. Injury to $\mathrm{MM}$ is the most common injury in knee joint trauma. In our study bone bruise is associated with decreased risk of MM tear. Appearance of bone bruise in knee MRI may imply that the force of trauma is absorbed by bone tissue. We hypothesize that such absorption may decrease the intensity of knee joint rotation. This restricted rotation may decrease risk of MM tear. Our result is in contrast with the findings of Bretlau et al. ${ }^{12}$ and Jelić et al. ${ }^{16}$ which showed that bone bruise is associated with increased risk of menisci injury and also is in contrast with the study of Vincken et al. ${ }^{17}$ which showed that bone bruise has not any association with injury to MM. As Table 1 shows, bone bruise is associated with increased risk of $\mathrm{ACL}$ and $\mathrm{MCL}$ tear. Bertula et al. ${ }^{12}$ and Vincken et al. ${ }^{17}$ found similar result.

Fracture. We found that existence of fracture around knee joint is associated with decreased risk of the tearing of $\mathrm{ACL}$ and MM but has not any association with tearing of other ligaments and LM. Occurrence of fracture around knee joint may absorb the force of trauma. We hypothesize that such absorption may decrease the intensity of knee joint rotation. This restricted rotation may decrease risk of $\mathrm{MM}$ tear. Absorption of the force of trauma in fracture may be 

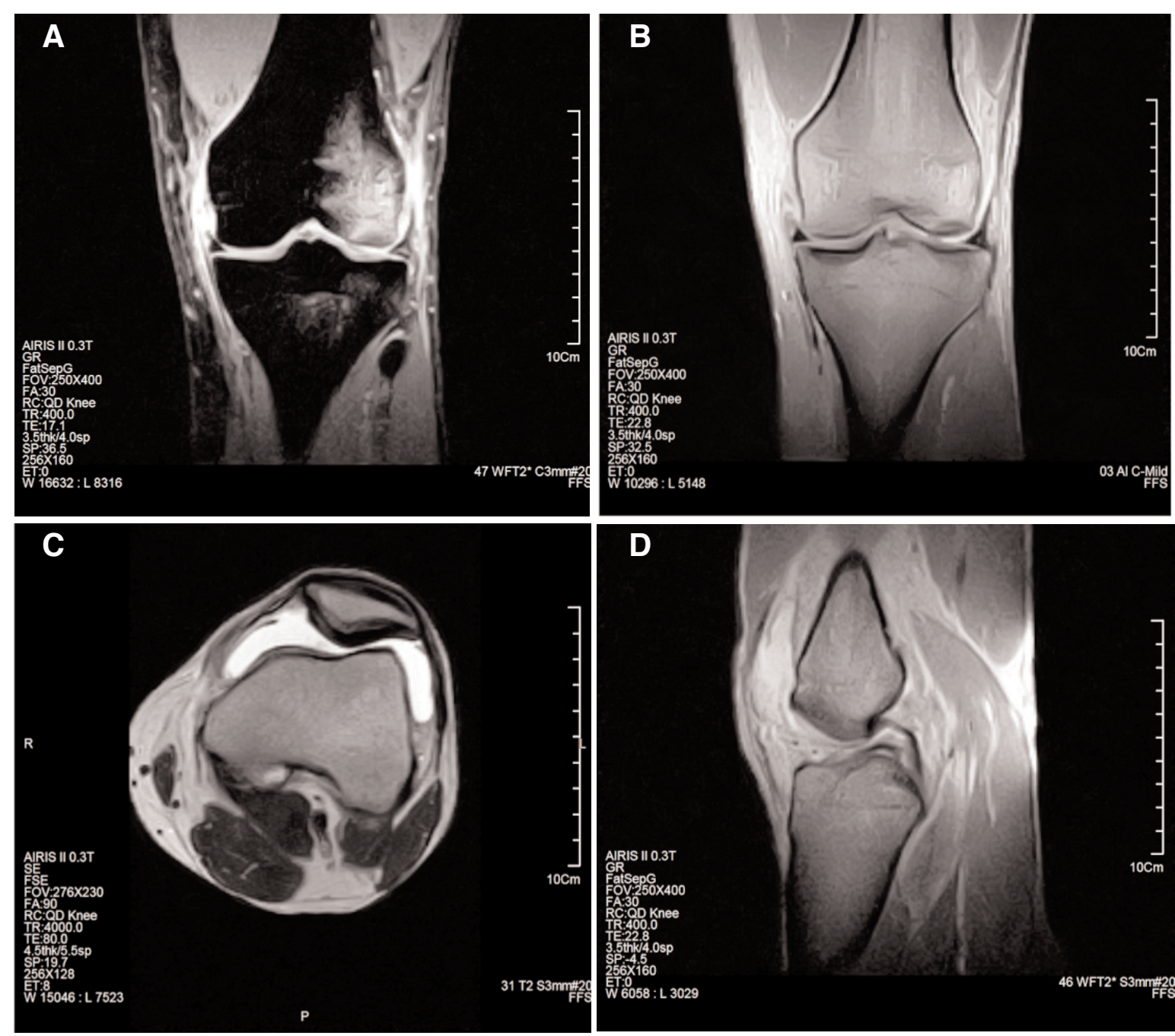

Figure 1. A 21-year-old male patient with knee trauma. A. Coronal gradient-echo (GE) fat suppressed image shows bone bruise at lateral femoral condyle and lateral tibial plateau as well as turn of MCL. B. Coronal GE in phase T2weighted image shows normal MM. C. Axial spin-echo (SE) T2 weighted image shows joint effusion. D. Sagittal GE in phase T2-weighted image shows complete tearing of ACL.

more than the absorption of the force of trauma in bone bruise. As Table 1 and Table 2 show, the difference of having MM tear between the group with bone bruise and group with non bone bruise is much less than the difference of having MM tear between the group with fracture and group without fracture. Also none of 21 patients with fracture had MM tear. Absorption of the force of trauma may also decrease the risk of $A C L$ tear.

Joint effusion. As Table 3 shows, only $A C L$ and MCL tear are significantly associated with pathologic joint effusion. Intracapsular inflammation after injury to ACL may explain the positive association between ACL tear and joint effusion. The association of joint effusion with $\mathrm{MCL}$ tearing may be related to anatomical proximity of MCL to capsule of knee joint. Also this association may be related to coincidence of $A C L$ and MCL tears. The association between MCL tear and joint effusion didn't loss its significance after statistical controlling of the ACL tear. So we conclude that MCL tear may affect the capsule too and induce intracapsular inflammatory response. There isn't any study about association between joint effusion and tearing of menisci and ligaments in acute knee trauma.

Our study has a limitation. Because of small sample size we could not do statistical analysis separately for partial and complete tear of cruciate and collateral ligament. Further study with larger sample size may reveal any difference between the groups with partial and complete tear.

The findings of this study may be helpful in reporting of the MRI of patients with knee trauma as follow: if there is bone bruise, MCL should be evaluated for any tearing more precisely. And if there is a joint effusion then ACL and MCL should be examined more 

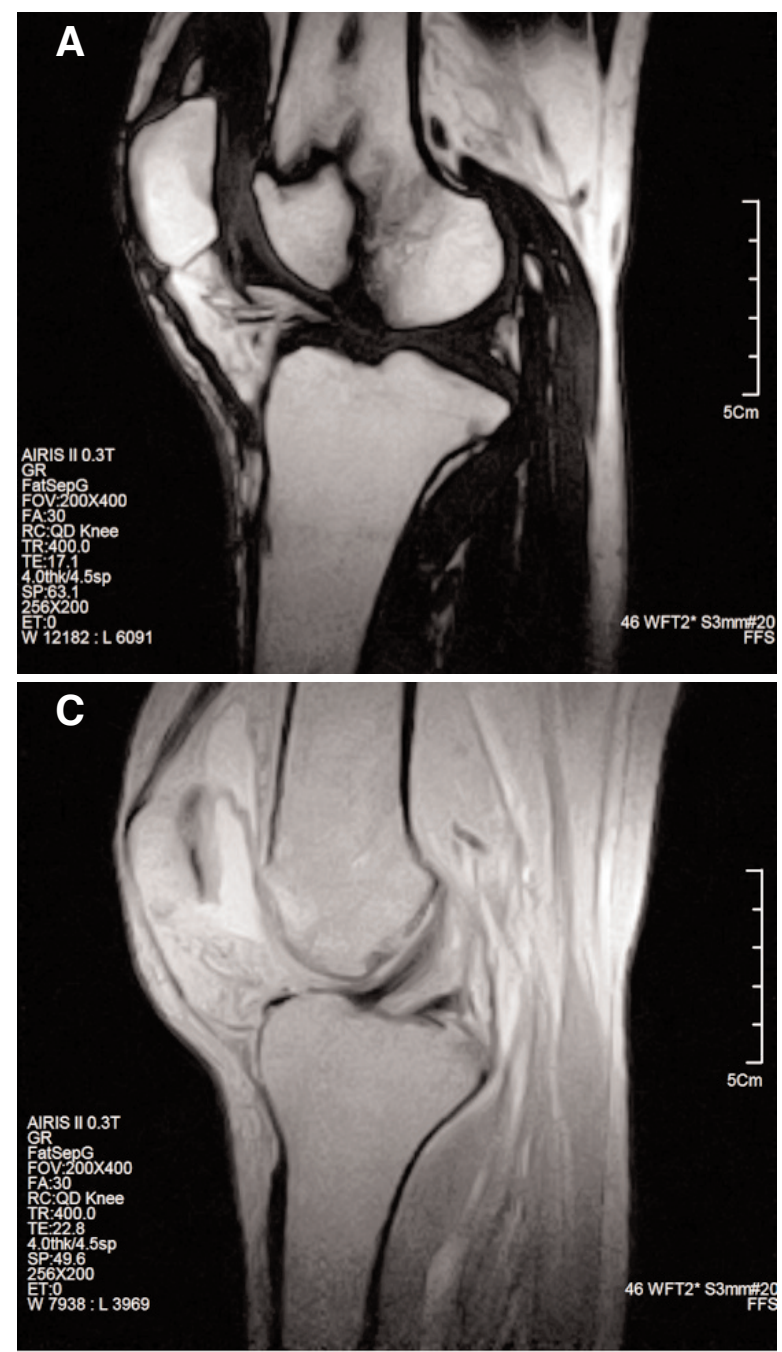

precisely. Also a radiologist may consider these two points in interpretation of knee MRI in patients with knee trauma: existence of bone bruise or fracture decreases the risk of the MM injury considerably and in patients with fracture around knee joint the risk of $\mathrm{ACL}$ injury is lower than patients without fracture.

\section{Conflict of interests}

The Authors declare that they have no conflict of interests regarding the publication of this paper.

\section{References}

1. Espeland A, Natvig NL, Løge I, Engebretsen L, Ellingsen J. Magnetic resonance imaging of the knee in Norway 20022004 (national survey): rapid increase, older patients, large geographic differences. BMC Health Serv Res. 2007;7:115.

2. Oei EH, Ginai AZ, Hunink MG. MRI for traumatic knee injury: a review. Semin Ultrasound CT MR. 2007;28:141-157.

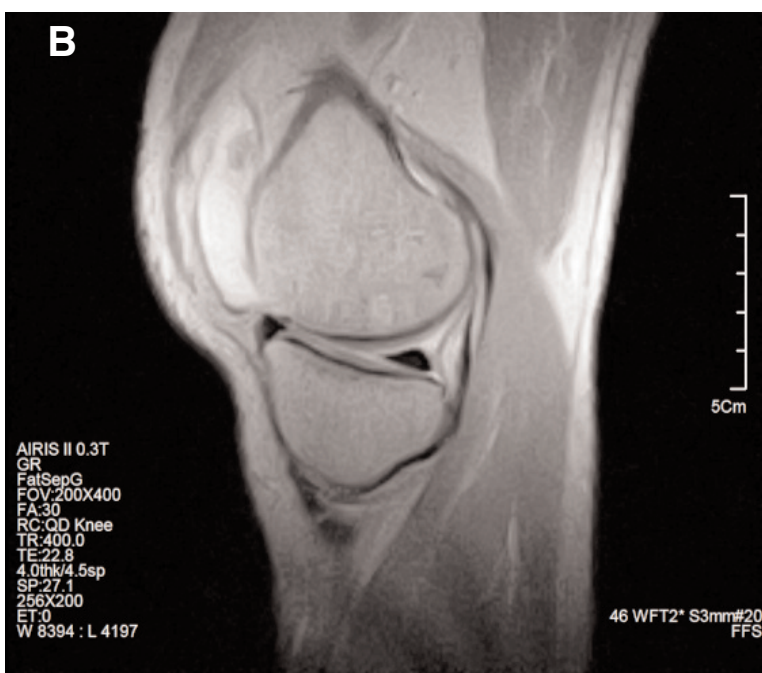

Figure 2. A 37-year-old male patient with knee trauma. A. Sagittal section fluid suppressed GE sequence shows a fracture at anterior aspect of lateral femoral condyle which is extend to joint surface. B. Sagittal section from MM in phase GE T2-weighted image shows normal MM. C. Sagittal section from ACL in phase GE T2-weighted image shows intact ACL.
3. Wörtler K. MR Imaging of the Knee. Radiologe. 2007;47:11311143.

4. Sanders TG, Miller MD. A systematic approach to magnetic resonance imaging interpretation of sports medicine injuries of the knee. Am J Sports Med. 2005;33:131-148.

5. Crawford R, Walley G, Bridgman S, Maffulli N. Magnetic resonance imaging versus arthroscopy in the diagnosis of knee pathology, concentrating on meniscal lesions and ACL tears: a systematic review. Br Med Bull. 2007;84:5-23.

6. Maurer EJ, Kaplan PA, Dussault RG, Diduch DR, Schuett A, McCue FC, et al. Acutely injured knee: effect of MR imaging on diagnostic and therapeutic decisions. Radiology. 1997;204: 799-805.

7. Stevens KJ, Dragoo JL. Anterior cruciate ligament tears and associated injuries. Top Magn Reson Imaging. 2006;17:347362.

8. Kapelov SR, Teresi LM, Bradley WG, Bucciarelli NR, Murakami DM, Mullin WJ, et al. Bone contusions of the knee: increased lesion detection with fast spin-echo MR imaging with spectroscopic fat saturation. Radiology. 1993;189:901-904.

9. Blin D, Cyteval C, Kamba C, Blondel M, Lopez FM. Imaging of traumatic injuries of the knee: J Radiol. 2007;88:775-788.

10. Yao L, Lee JK. Occult intraosseous fracture: detection with MR imaging. Radiology. 1988;167:749-751. 
11. Newberg AH, Wetzner SM. Bone bruises: their patterns and significance. Semin Ultrasound CT MR. 1994;15:396-409.

12. Bretlau T, Tuxøe J, Larsen L, Jørgensen U, Thomsen HS, Lausten GS, et al. Bone bruise in the acutely injured knee. Knee Surg Sports Traumatol Arthrosc. 2002;10:96-101.

13. Nakamae A, Engebretsen L, Bahr R, Krosshaug T, Ochi M. Natural history of bone bruises after acute knee injury: clinical outcome and histopathological findings. Knee Surg Sports Traumatol Arthrosc. 2006;14:1252-1258.

14. Johnson MW. Acute knee effusions: a systematic approach to diagnosis. Am Fam Physician. 2000;61:2391-2400.

15. Padulo J, Oliva F, Frizziero A, Maffulli N. Muscles, Ligaments and Tendons Journal. Basic principles and recommendations in clinical and field science research. MLTJ. 2013;3 (4):250-252.

16. Jelić $D$, Masulović $D$. Bone bruise of the knee associated with the lesions of anterior cruciate ligament and menisci on magnetic resonance imaging. Vojnosanit Pregl. 2011;68:762-766.

17. Vincken PW, Ter Braak BP, van Erkel AR, Coerkamp EG, Mallens WM, Bloem JL. Clinical consequences of bone bruise around the knee. Eur Radiol. 2006;16:97-107. 